\title{
Indoor PM10 Upregulates Pro-inflammatory Mediators in Bronchial Epithelial Cells
}

\author{
Chongxu Zhang ${ }^{1}$, Huda Asif ${ }^{2}$, Tess M Calcagno ${ }^{3}$, Naresh Kumar ${ }^{4}$, Nevis L Fregien ${ }^{5}$, and Mehdi \\ Mirsaeidi ${ }^{1,2,4^{*}}$ \\ 1 Section of Pulmonary, Miami VA Healthcare System, Miami, FL \\ 2 Division of Pulmonary and Critical Care, University of Miami, Miami, FL \\ 3 School of Medicine, University of Miami, Miami, FL \\ 4 Department of Public Health, University of Miami, Miami, FL \\ 5 Department of Biology, University of Miami, Miami, FL \\ * Correspondence: e-mail: msm249@med.miami.edu
}

\begin{abstract}
Indoor dusts are collectively formed from anthropogenic and atmospheric activities. Particle matter $10\left(\mathrm{PM}_{10}\right)$ is inhalable and causes significant inflammation by interaction with the pulmonary epithelial barrier. The mediators involved in bronchial epithelial cells response to dust are remined unknown. The air-liquid interface of our lung on chip model was exposed to indoor dust collected from highly polluted houses in Delhi, India. The media were collected after 4 days and cytokine levels were measured. We found that the concentration of IFN $\alpha$, IFN $\gamma$, Interleukin-6 (IL-6), IL1b, TNFa, and Granulocyte monocyte colony stimulating factor (GM-CSF) were significantly increased after exposure to indoor dust. IFN type I pathways were a major response from dust exposure. Further investigation is needed to determine the mechanism of action and targets of dust in bronchial epithelial cells.
\end{abstract}

Keywords: Indoor, $\mathrm{PM}_{10}$; pulmonary disease; inflammation; IFN; type I interferon; cytokine; epithelial cell

\section{Introduction}

Indoor exposure to aerosolized particulate matter up to ten micrometers in size ( $\left.\mathrm{PM}_{10}\right)$ is a significant respiratory health concern, especially in urban environments and in low-middle income countries with poor air quality [1]]. Indoor PM10 may come from indoor activities like cooking, or it may be transported from outdoor air pollution [1, 2]. In fact, PM10 is spatially and temporally correlated to outdoor anthropogenic activity [2]. During inspiration, PM10 is brought into the respiratory tract through airflow convection. Given its size, $\leq 10 \mu \mathrm{m}$ in aerodynamic diameter, $\mathrm{PM}_{10}$ deposits in bronchial airways through sedimentation or inertial impaction leading to the subsequent development of pulmonary disease [3]. The link between increased air pollution and the development of pulmonary pathology is attributed to direct oxidant release from organic and metal components as well as indirect oxidative cellular responses. Production of reactive oxygen species and oxidative stress initiates an inflammatory cascade leading to cellular apoptosis [4]. PM10 penetrates the mucosal layer of bronchial airways leading to localized epithelial damage which is subsequently amplified by cytokine production [ $[$ ].

Interferon- $\alpha$ is a type I interferon (IFN 1) implicated in regulation of inflammation in viral infections and immune disorders [6]. Recently, we investigated the role of Mycobacterial cell wall microparticles on normal human bronchial epithelial (NHBE) cells and found a differential 
overexpression of IFN 1 pathway proteins and genes in the exposed NHBE cells as compared to unexposed cells suggesting an innate IFN I mediated immune response in NHBE [7]. We hypothesized that IFN I could also have a central role in mediation of innate immune response in NHBE cells following exposure to indoor $\mathrm{PM}_{10}$. Literature review revealed no previous study on the role of the IFN I pathway in bronchial epithelial responses to respirable PM10 exposure. In this study, we explored the role of IFN 1 in NHBE cells following exposure to PM10 dust in a highly polluted urban setting.

\section{Materials and Methods}

Indoor PM10 was collected from a residential setting (patient houses) in Delhi, India on $37 \mathrm{~mm}$ PTFE membrane filters using a vacuum pump for 72 hours. The samples were analyzed using Kevex $^{\mathrm{TM}}$ Energy Dispersive X-Ray Spectrometer to identify elemental composition of $\mathrm{PM}_{10}$ at Environmental Protection Agency (EPA)'s National Exposure Research Laboratory, Research Triangle Park, NC.

Extracted PM10 dust was then exposed to NHBE in the lung-on-chip model (LOCM). LOCM is a biological lung model designed to simulate a physiological human airway with two cell line layers; primary NHBE isolated from lungs rejected for transplant at University of Miami at the top of the membrane and endothelial cells (Human Lung Microvascular Endothelial Cells, Lonza, Walkersville, $\mathrm{MD}$ ) at the bottom. A transwell ${ }^{\circledR}$ polyester membrane cell culture insert (12 mm diameter, $0.4 \mu \mathrm{m}$ pore size; Corning Life Sciences, Amsterdam, The Netherlands) was used for cell culturing as discussed elsewhere $[\underline{7}, \underline{8}]$.

An air channel was connected to the epithelial layer while a media channel was connected to the endothelial layer as shown in Figure 1. PM10 exposure was set to a concentration of $500 \mathrm{ppm}$ in a $55.7 \mu \mathrm{L}$ solution to $944.3 \mu \mathrm{L}$ of media and was added to ALI air-liquid interface. After 4 days of exposure, the medium was collected and cytokines (IL-1RA, IL-2, , IL-2R, IL-4, IL-5, IL-6, IL-7, IL8,IL-10, IL-12 (p40/p70), IL-13, IL-15, IL-17, GM-CSF, Eotaxin, IFN- $\alpha$, IP-10, IFN- $\gamma$, MCP-1, IL-1 $\beta$, MIG, MIP- $1 \alpha$, , MIP-1 $\beta$, RANTES, TNF- $\alpha$ ) were measured using Human Magnetic 25-Plex Kit (Thermofisher, Catalog number: LHC0009M). Data were reported as mean \pm standard error (SE), and each experiment was repeated 3 times. A paired $t$-test was used to compare two means. A $p<0.05$ was defined as statistically significant. The String dataset was used to determine the protein-protein interaction (PPI) networks [9]. The PPI was limited to "Homo sapiens" and interaction score more than 0.4 were used to develop the network.

\section{Results}

The indoor dust sample had a concentration of $315078 \mathrm{ng} / \mathrm{m} 3$ with $64.97 \%$ of the PM10 containing various elemental species. The ten most abundant elements found in the sample were silicon $(34.72 \%)$, iron (12.50\%), aluminum (12.49\%), calcium (12.11\%), potassium $(8.79 \%)$, sulfur $(5.67 \%)$, lead $(4.89 \%)$, zinc $(3.96 \%)$, chloride $(2.36 \%)$, and magnesium $(2.54 \%)$. In the LOCM experiment, the collected media showed a statistically significant increase in IFN $\alpha$, IFN $\gamma$, Interleukin-6 (IL-6), IL1 $\beta$, $\mathrm{TNF} \alpha$, and Granulocyte monocyte colony stimulating factor (GM-CSF) in LOCM exposed to PM10 as compared to control as shown in Figure 1. The rest of cytokines were not significantly changed between two groups. In the PPI network, IFN $\alpha$ functionality is associated with TNF $\alpha$, IL6, IFN $\gamma$, and GM-CSF as depicted in Figure 1. 


\subsection{Figures}
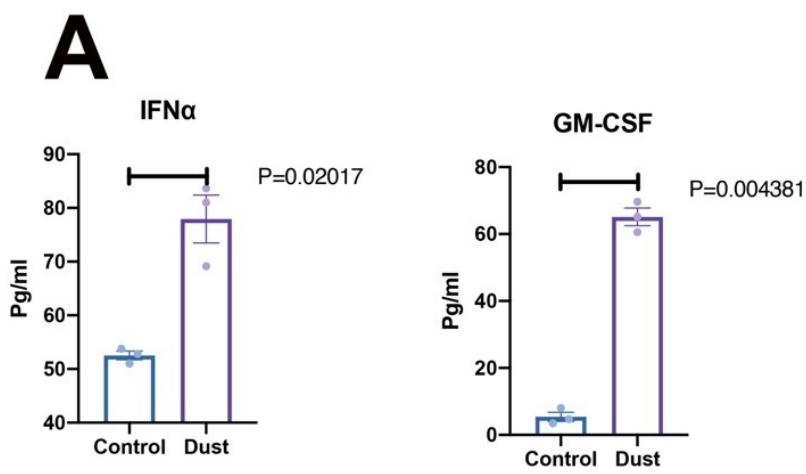

B
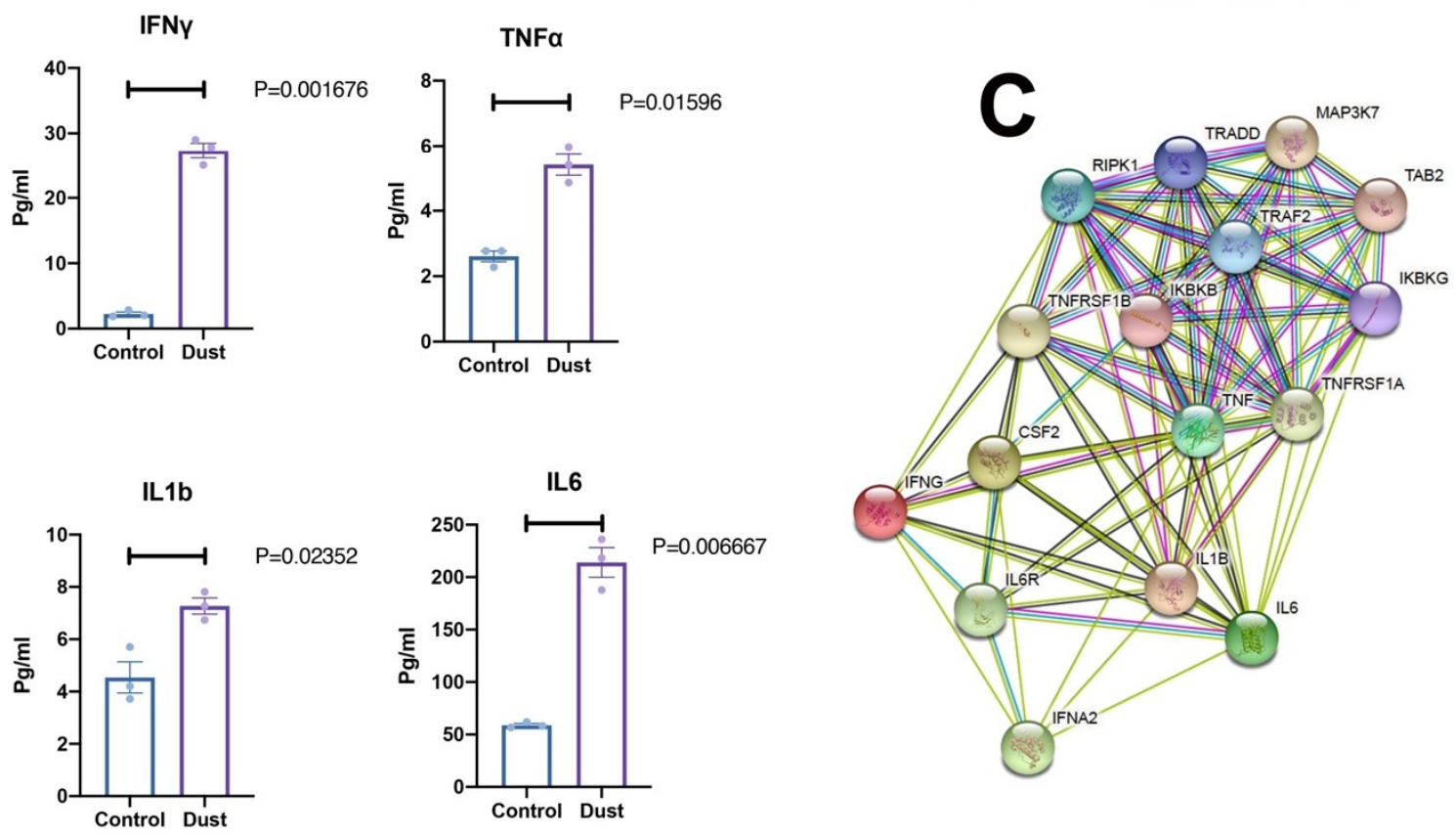

Figure 1. (a) Shows cytokines with significant expressions in media after exposure of ALI with indoor PM10. Dust (PM10) was exposed to the ALI of lung model as shown in (b). Top layer is mature pseudostratified bronchial epithelial cells, membrane in the middle and pulmonary small vessels endothelial in the bottom of the membrane. (c) Shows String interaction network of cytokines found with significant increase in PM10 exposure and their first level of protein interaction in the network. 


\section{Discussion}

This study found a statistically significant increase in multiple cytokines in a lung-on-chip model after exposure to indoor particles composed of various chemical elements (most commonly silicon). Specifically, INF- $\alpha$ concentrations increased relative to non-exposed lung models $(\mathrm{p}<0.02)$ along with an associated increase in other cytokines (IFN $\gamma$, IL-6, TNF- $\alpha$, and GM-CSF) suggesting a complex immune response involving the interplay between multiple related cytokines.

IFN $\alpha$ is produced by almost all body cells including epithelial cells. IFN $\alpha$ is known as a moving target due its ability to mediate opposing effects [10]. While IFN $\alpha$ demonstrates an anti-inflammatory role in virally infected epithelium and multiple sclerosis, it is also the potential orchestrator of pathological inflammation in certain immune disorders such as systemic lupus erythematosus []ㅡ. Its dichotomous role in inflammation is explained by its opposing effects on helper T cell subtypes, TH1 and TH2. Previous studies show IFN $\alpha$ can enhance TH1 response via activation of inflammatory cytokines including IL12, IL18 and caspase1 and inhibit TH2 via activation of IL4 [11, $\underline{12}]$.

TH1 mediated response is further reinforced by TH1 secreted IFN $\gamma$ which increases naïve T-cell responsiveness to IL12 [12]. Our study revealed elevated levels of IFN $\gamma(p<0.001)$, suggesting a complementary role of IFN $\gamma$ in propagation of TH1 response.

There is evidence suggesting that IFN $\alpha$ mediates an increases in IL6, a cytokine which was elevated in our study $(\mathrm{p}<0.01)$, in the setting of toll-like receptor (TLR) 8 activation in neutrophils [ $\underline{13}$ ]. TLR8 is an endosomal receptor known to recognize single-stranded DNA viral proteins[13]. Given that TLR8 are also present in bronchial epithelial cells suggests a possible mechanism of IL6 activation via IFNa. Furthermore, the process of IL6 activation is potentiated by the TH1 committed proinflammatory cytokine tumor necrotizing factor (TNF) $\alpha$, which was also elevated in our study $(\mathrm{p}<0.01)[\underline{13}]$.

Our proposed mechanism for the sequence of cytokine release is listed as the following. Indoor aerosolized particle matter first deposits in bronchial airways. IFN $\alpha$ is produced by bronchial epithelial cells which leads to TH1 activation and TH2 inhibition. Activation of TH1 cells leads to the subsequent release of cytokines IFN $\gamma$ and $\operatorname{TNF} \alpha$. IFN $\gamma$ further increases naive TH1 cell activation and mediates increases in IL6 via action of TLR 8 found in bronchial epithelial cells. Similarly, TNF $\alpha$ potentiates IL6 signaling.

The mechanism of $\mathrm{PM}_{10}$ induced inflammation is largely still unknown, but the inflammatory host response to aerosolized particulate matter has been previously studied. Bossmann $e t$ al. studied the inflammatory response in peripheral blood mononuclear cells after exposure to PM10 particles and found notable increases in TNF $\alpha$, IL-6, and IL-1 $\beta[14]$. Park et al. studied human dermal fibroblast response to PM10 and observed increases in IL-1 $\beta$, IL-6, IL-8 and IL33 [15]. Becker et al. found that alveolar macrophage response to virally infected lung epithelial cells was altered in response to PM10 exposure [16]. Ramage et al. found increased expression of C-reactive protein and heat-shock protein70 secondary to oxidative inflammatory response in lung epithelial cells after treatment with PM10 [17].

Recently, we found overexpression of IFN1 proteins in NHBE cells in response to mycobacteria cell wall particles, which prompted the development of this study to look for a similar response in the setting of $\mathrm{PM}_{10}$ exposure [7]. This study was the first to demonstrate a significant proinflammatory IFN $\alpha$ mediated inflammatory cascade in response to PM10. However, further studies are needed to identify the presence of TH1 cell lines, mRNA expression of cytokines, and 
gene/protein expression of cytokines to fully elucidate our proposed mechanism. Content of the PM10 was analyzed for the percent component of each chemical element, but individual chemical elements were not assessed for biological response. Additional studies are needed to assess the biological response of each component to increase clinical applicability.

Author Contributions: Conceptualization, M.M.; methodology, C.Z, N.F, N.K.; formal analysis, C.Z, N.F, N.K, M.M.; investigation, C.Z, N.F,N.K.; writing-original draft preparation, HA.; writing - review and editing, T.C.; All authors have read and agreed to the published version of the manuscript.

Funding: None

Acknowledgments: Authors would like to thank Dr. Greg Holt (University of Miami) for his critical comments on the manuscript.

Conflicts of Interest: All authors disclose no conflict of interest. 


\section{Appendix B}

All appendix sections must be cited in the main text. In the appendixes, Figures, Tables, etc. should be labeled starting with 'A', e.g., Figure A1, Figure A2, etc.

\section{References}

1. Abt E, Suh HH, Catalano P, Koutrakis P: Relative contribution of outdoor and indoor particle sources to indoor concentrations.(Brief Article). Environmental Science \& Technology 2000, 34:3579.

2. Elbayoumi M, Ramli NA, Yusof NFFM: Spatial and temporal variations in particulate matter concentrations in twelve schools environment in urban and overpopulated camps landscape. Building and Environment 2015, 90:157.

3. Gutierrez MP, Zuidema P, Mirsaeidi M, Campos M, Kumar N: Association between African Dust Transport and Acute Exacerbations of COPD in Miami. J Clin Med 2020, 9.

4. Ghio AJ, Carraway MS, Madden MC: Composition of air pollution particles and oxidative stress in cells, tissues, and living systems. J Toxicol Environ Health B Crit Rev 2012, 15:1-21.

5. Falcon-Rodriguez CI, Osornio-Vargas AR, Sada-Ovalle I, Segura-Medina P: Aeroparticles, Composition, and Lung Diseases. Frontiers in immunology 2016, 7:3-3.

6. Kalliolias GD, Ivashkiv LB: Overview of the biology of type I interferons. Arthritis Res Ther 2010, 12 Suppl 1:S1.

7. Zhang C, Asif H, Holt GE, Griswold AJ, Campos M, Bejarano P, Fregien NL, Mirsaeidi M: Mycobacterium abscessus-Bronchial Epithelial Cells Cross-Talk Through Type I Interferon Signaling. Front Immunol 2019, 10:2888.

8. Korukonda A, Zhang C, Rodriguez L, Guerrero A, Campos M, Holt G, Fregien NL, Conner G, Mirsaeidi M: Electronic Cigarettes Enhance Replication of Mycobacterium abscessus in Airway Epithelial Cells. Am J Respir Cell Mol Biol 2019, 60:717-719.

9. Szklarczyk D, Gable AL, Lyon D, Junge A, Wyder S, Huerta-Cepas J, Simonovic M, Doncheva NT, Morris JH, Bork P, et al: STRING v11: protein-protein association networks with increased coverage, supporting functional discovery in genome-wide experimental datasets. Nucleic Acids Res 2019, 47:D607-D613.

10. Rizza P, Moretti F, Belardelli F: Recent advances on the immunomodulatory effects of IFNalpha: implications for cancer immunotherapy and autoimmunity. Autoimmunity 2010, 43:204-209.

11. Jarry A, Malard F, Bou-Hanna C, Meurette G, Mohty M, Mosnier JF, Laboisse CL, Bossard C: Interferon-Alpha Promotes Th1 Response and Epithelial Apoptosis via Inflammasome Activation in Human Intestinal Mucosa. Cell Mol Gastroenterol Hepatol 2017, 3:72-81.

12. Wenner CA, Guler ML, Macatonia SE, O'Garra A, Murphy KM: Roles of IFN-gamma and IFN-alpha in IL-12-induced T helper cell-1 development. J Immunol 1996, 156:1442-1447.

13. Zimmermann M, Arruda-Silva F, Bianchetto-Aguilera F, Finotti G, Calzetti F, Scapini P, Lunardi C, Cassatella MA, Tamassia N: IFNalpha enhances the production of IL-6 by human neutrophils activated via TLR8. Sci Rep 2016, 6:19674.

14. Bossmann K, Bach S, Höflich C, Valtanen K, Heinze R, Neumann A, Straff W, Süring K: Holi colours contain PM10 and can induce pro-inflammatory responses. J Occup Med Toxicol 2016, 11:42. 
15. Park SY, Byun EJ, Lee JD, Kim S, Kim HS: Air Pollution, Autophagy, and Skin Aging: Impact of Particulate Matter (PM(10)) on Human Dermal Fibroblasts. Int J Mol Sci 2018, 19.

16. Becker S, Soukup JM: Exposure to urban air particulates alters the macrophage-mediated inflammatory response to respiratory viral infection. J Toxicol Environ Health A 1999, 57:445457.

17. Ramage L, Guy K: Expression of C-reactive protein and heat-shock protein-70 in the lung epithelial cell line A549, in response to PM10 exposure. Inhal Toxicol 2004, 16:447-452. 